\title{
HOPON (Hyperbaric Oxygen for the Prevention of Osteoradionecrosis): a randomised controlled trial of hyperbaric oxygen to prevent osteoradionecrosis of the irradiated mandible: study protocol for a randomised controlled trial
}

Richard Shaw ${ }^{1 *}$ (D), Christopher Butterworth', Binyam Tesfaye ${ }^{2}$, Matthew Bickerstaff², Susanna Dodd ${ }^{3}$, Gary Smerdon ${ }^{4}$, Seema Chauhan ${ }^{2}$, Peter Brennan ${ }^{5}$, Keith Webster ${ }^{6}$, James McCaul ${ }^{7}$, Peter Nixon ${ }^{8}$, Anastasios Kanatas ${ }^{8}$ and Paul Silcocks ${ }^{2}$

\begin{abstract}
Background: Osteoradionecrosis of the mandible is the most common serious complication of radiotherapy for head and neck malignancy. For decades, hyperbaric oxygen has been employed in efforts to prevent those cases of osteoradionecrosis that are precipitated by dental extractions or implant placement. The evidence for using hyperbaric oxygen remains poor and current clinical practice varies greatly. We describe a protocol for a clinical trial to assess the benefit of hyperbaric oxygen in the prevention of osteoradionecrosis during surgery on the irradiated mandible.

Methods/design: The HOPON trial is a phase III, randomised controlled, multi-centre trial. It employs an unblinded trial design, but the assessment of the primary endpoint, i.e. the diagnosis of osteoradionecrosis, is assessed on anonymised clinical photographs and radiographs by a blinded expert panel. Eligibility is through the need for a high-risk dental procedure in the mandible where at least 50-Gy radiotherapy has been received. Patients are randomised 1:1 to hyperbaric oxygen arm (Marx protocol) : control arm, but both groups receive antibiotics and chlorhexidine mouthwash. The primary endpoint is the presence of osteoradionecrosis at 6 months following surgery, but secondary endpoints include other time points, acute symptoms and pain, quality of life, and where implants are placed, their successful retention.
\end{abstract}

Discussion: The protocol presented has evolved through feasibility stages and through analysis of interim data. The classification of osteoradionecrosis has undergone technical refinement to ensure that robust definitions are employed. The HOPON trial is the only multi-centre RCT conducted in this clinical setting despite decades of use of hyperbaric oxygen for the prevention of osteoradionecrosis.

Trial registration: European Clinical Trials Database, ID: EudraCT200700622527. First registered on 5 November 2007.

\footnotetext{
* Correspondence: rjshaw@liv.ac.uk

${ }^{1}$ CRUK Liverpool Cancer Trials Unit, Department of Molecular and Clinical

Cancer Medicine, University of Liverpool and Aintree University Hospital NHS

Foundation Trust, Liverpool, UK

Full list of author information is available at the end of the article
} 


\section{Background}

Osteoradionecrosis (ORN) describes the process where irradiated bone undergoes necrosis and becomes exposed through the investing soft tissues for a period of at least 3 months [1-3]. An important precipitating factor for mandibular ORN is surgical trauma, commonly dental extractions or implant placement following head and neck radiotherapy, but ORN can also occur spontaneously. ORN is painful and debilitating, often requiring surgical resection of the jaw and complex multidisciplinary management [4]. The morbidity and mortality of ORN is significant and treatment outcomes often unsatisfactory. Post-radiation extractions should, self-evidently, be performed atraumatically and antibiotics are commonly prescribed [5], but there is a paucity of high-quality evidence to guide best practice in the prevention of ORN.

Preventive approaches include pre-radiotherapy extraction of teeth and the use of hyperbaric oxygen (HBO) treatments or prophylactic antibiotics for postradiotherapy extractions [6]. The overall incidence of ORN among post-radiotherapy patients is not certain but may have declined with improvement of radiotherapy techniques including intensity-modulated radiotherapy (IMRT) over recent decades [7]. However, the incidence of head and neck cancer, the proportion of cases receiving radiotherapy, and prognosis are all increasing [8], contributing to an expansion in the 'at risk' population where ORN prevention must be addressed. The risk of ORN with dental extractions is higher in the posterior mandible, with radiotherapy doses higher than 60 Gy (or with brachytherapy) and in smokers [9].

There have only been limited trials of prophylactic HBO. The single-centre randomised controlled trial (RCT) by Marx et al. [10] showed a significantly lower incidence of ORN after post-radiotherapy dental extractions in the HBO group when compared with the control group. There were two cases of ORN in 37 patients (5.4\%) receiving $\mathrm{HBO}$ undergoing 156 extractions, compared with 11 cases in 37 patients (29.9\%) undergoing 135 extractions receiving prophylactic penicillin, resulting in a number needed to treat (NNT) of 4 . In a nonrandomised retrospective study, Vudiniabola et al. [11] showed that of 29 patients who received pre-extraction HBO, 1 (3.4\%) developed ORN; and of 7 patients who did not receive HBO, 1 (14.3\%) developed ORN. Despite improvements in radiotherapy since the time of these studies, and evidence of a decreasing incidence of ORN [12], prophylactic HBO to prevent ORN has remained a standard of care for high-risk dental extractions [13].

$\mathrm{HBO}$ is not available to all patients as it requires proximity to a chamber, it is costly (approximately $£ 4000-6000$ per course) and sometime logistically problematic, requiring multiple daily visits. A typical protocol for prevention dictates $45 \mathrm{~h}$ of treatment over 30 compressions [10]. There are also risks associated with HBO therapy. A comprehensive recent safety review [14] noted common temporary visual problems, Eustachian tube problems $2 \%$, claustrophobia $2 \%$ and seizure $<0.01$.

A survey of UK practice revealed that, in a high-risk extraction of a lower mandibular molar, 33\% 'never', $41 \%$ 'sometimes' and 26\% 'usually' or 'always' prescribe prophylactic HBO [15]. Questionnaires of attitude to RCTs for HBO revealed that $93 \%$ of responders would wish to recruit such patients into multi-centre RCT [16]. Additionally, there is a lack of agreement on $\mathrm{HBO}$ protocol, with some UK patients referred for treatment in centres using non-standard pressures [17], lower than the typical 2.4 atmospheres (ATA) recommended in most ORN publications. Current National Institute for Health and Care Excellence (NICE) guidelines on the management of ORN recommend the use of $\mathrm{HBO}$ only as part of a clinical trial [18].

An additional question regarding the efficacy of $\mathrm{HBO}$ surrounds the placement of osseointegrated implants into the irradiated mandible, with the aim of preventing ORN or optimising bony healing and implant retention. Survey data again suggest wide disparity in practice [15] in the UK. No RCT has been carried out in this setting, and there are conflicting data from retrospective case control series $[19,20]$ as to whether $\mathrm{HBO}$ may enhance survival of implants in irradiated bone.

\section{Objective}

The objective of the HOPON trial is to determine the benefit of $\mathrm{HBO}$ in the prevention of ORN subsequent to a surgical procedure in the 'at risk' irradiated mandible. The procedures included are high-risk dental extractions and the placement of implants. Additional outcomes are to document the incidence of ORN in the control group, the outcomes where ORN is diagnosed, and the retention of implants. The trial will also measure the effect of $\mathrm{HBO}$ in post-operative pain and effects on quality of life. The HOPON phase III trial protocol described here has evolved from the feasibility objectives in a preceding HOPON feasibility study.

\section{Methods/Design \\ Design}

HOPON is a prospective, multi-centre, randomised controlled, phase III trial to assess the effectiveness of preventing ORN after surgical procedures in the irradiated mandible. Although the patients and investigators are unblinded, the primary endpoint is assessed by a blinded panel for the presence and grade of ORN. Other endpoints are unblinded as they are recorded by either the patients or site investigators. 


\section{Study population and eligibility}

Patients who meet the eligibility criteria (Table 1 ) will be recruited either from routine review following the treatment of head and neck cancer, or following referral for oral rehabilitation. Detailed information on the benefits and risks of the study will be provided to the patients, including the provision of $\mathrm{HBO}$ at the nearest suitable trial chamber.

\section{Randomisation and blinding}

Patients who meet eligibility criteria and have given informed consent will be randomly assigned by the Cancer Research UK Liverpool Cancer Trials Unit (LCTU). Randomisation will be in a 1:1 ration between $\mathrm{HBO}$ arm and control (non-HBO) arm, stratified by recruiting centre. The randomisation code list will be generated by the LCTU trial statistician by means of block randomisation [21] with randomly varying block length. The clinical team will be informed of the allocation of each patient by fax. Allocation of treatment is unblinded to local investigators and patients. The trial schema is shown in Fig. 1.

\section{Trial intervention and control treatments}

The supplier of the oxygen to be used will be dependent on standard practice within each hyperbaric unit. Treatment will be administered based around a version of the Royal Navy Therapeutic Table 66 - Repeat Hyperbaric Oxygen Therapy [22]. The patient undergoes pressurisation to 2.4 ATA at a tolerable rate. The patient undergoes decompression after $100 \%$ oxygen has been breathed at 2.4 ATA for a total of between 80 and $90 \mathrm{~min}$. Air breaks while at 2.4 ATA may be introduced routinely or as required. The decompression is scheduled to control satisfactorily any risk to the patient and, if present, to the in-chamber attendant. Patients should breathe oxygen at an inspired partial pressure greater than 2.0 ATA for no more than 110 min during each individual treatment.

Patients in both arms of the trial are given chlorhexidine mouthwash and antibiotics. Pre- and post-operative chlorhexidine mouthwash $0.2 \%$ is used in a volume of
$10 \mathrm{ml}$ (i.e. one capful) washed around the mouth for around $1 \mathrm{~min}$ and spat out, three times daily (tds) for 5 days post-operatively. In case of chlorhexidine allergy, warm salt mouthwash at 1 teaspoon per cup of warm water is used. Orally administered antibiotics comprise amoxicillin $3 \mathrm{~g} 1 \mathrm{~h}$ pre-operatively (or $1 \mathrm{~g}$ administered intravenously) and $250 \mathrm{mg}$ tds for 5 days postoperatively. In case of penicillin allergy this will be: $600 \mathrm{mg}$ orally administered clindamycin (either 600-mg tablets orally (or the same dose of a $75-\mathrm{mg} / 5 \mathrm{ml}$ suspension if tablets not tolerated) given $1 \mathrm{~h}$ pre-operatively (or $600 \mathrm{mg}$ administered intravenously at the time of surgery) and $200 \mathrm{mg}$ metronidazole orally tds for 5 days post-operatively.

\section{Outcome measures}

The primary outcome measure is the diagnosis of ORN 6 months following surgery as determined by a blinded central review of clinical photographs and radiographs as well as the site investigator's assessment. This blinded review is carried out by an expert panel, with access to paired clinical photographs and radiographs (orthopantomogram). Additionally, where possible, any area of exposed bone is presented in context of an in-field rule and/or site investigator's measurement on the case report forms. The diagnosis of ORN at 3 and 12 months are secondary outcome measures, but determined in a similar way, the only difference being that radiographs will only be taken at these time points if ORN has been clinically diagnosed. A summary of the outcome measures and their time points is presented in Table 2.

The definition and classification of ORN used in the trial, based on that of Notani [23], has been subject to a technical refinement through protocol amendment following blinded analysis of the HOPON feasibility data [24], and is presented in Fig. 2. This was required to resolve inconsistencies which arose in cases where very small areas of exposed bone were seen: 'minor bone spicules' (MBS) were apparent, which occurred in 19\% of patients in this initial analysis. In reporting cases with MBS, some trial investigators took a pragmatic position in judging MBS as clinically unimportant, likely gradually healing, and not reflecting progressive ORN.

Table 1 Eligibility criteria

\begin{tabular}{ll}
\hline Inclusion criteria & Exclusion criteria \\
\hline Age $>18$ years & Prior diagnosis of ORN of the mandible \\
Prior radiotherapy to mandible $>50 \mathrm{~Gy}$ & Prior HBO therapy for any indication \\
No evidence of cancer recurrence & Any previous prescription of systemic bisphosphonates, pentoxyphylline or tocopherol \\
Condition requiring surgery to mandible: & Pregnancy \\
$\quad$ Extraction of premolar or molar & Contraindications to HBO: \\
Implant placement & Lung disease (severe COPD or bullae) \\
Provision of written informed consent in competent patient & Middle ear \\
& Claustrophobia \\
&
\end{tabular}

COPD chronic obstructive pulmonary disease, $H B O$ hyperbaric oxygen, $O R N$ osteoradionecrosis 


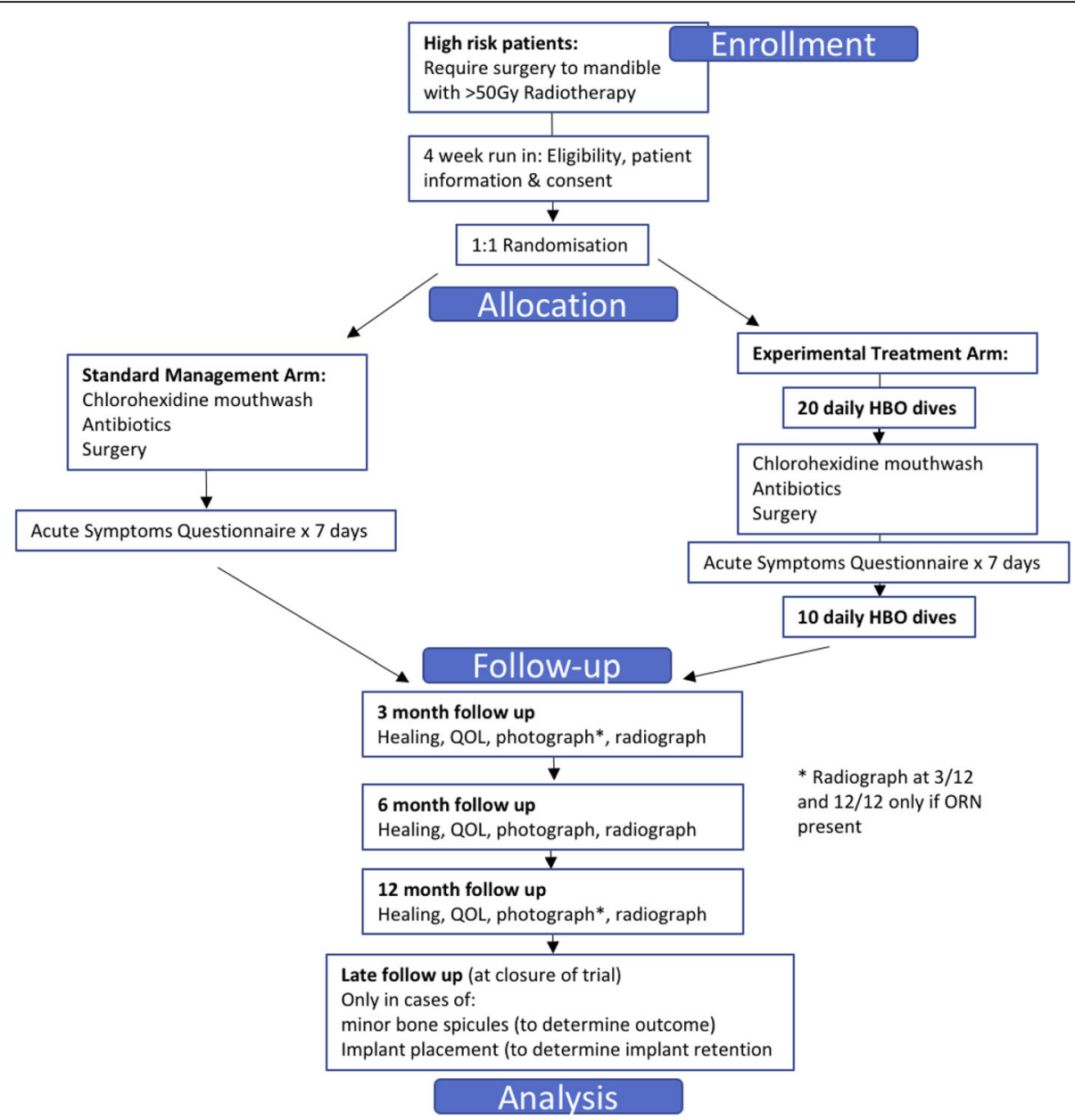

Fig. 1 Hyperbaric Oxygen for the Prevention of Osteoradionecrosis (HOPON) trial schema (Consolidated Standards of Reporting Trials (CONSORT) format)

Others classified as Notani 1 ORN based on rigid definitions in common clinical use. When MBS was added as an additional distinct category to the classification (as $<20 \mathrm{~mm}^{2}$ ) this ambiguity was resolved and agreement between observers was achieved. This refinement was adopted by the protocol after discussion with trial oversight committees and the relevant regulatory bodies. A working assumption was made, after consultation, to group MBS with the healed ('not ORN') cases, but another outcome measure of the trial will be to test this assumption against the outcomes for later time points. For the purpose of the HOPON trial, radiological signs alone, or pain alone, are not considered ORN unless accompanied by a breach in overlying skin or mucosa.

As the primary endpoint of the trial is dependent on the availability and quality of radiographs and clinical photographs, a quality-control protocol is adopted. All new centres recruiting patients will have the first sets of images audited, and then $10 \%$ thereafter. These will be scored as adequate/inadequate to assess the presence of ORN by an appropriate panel assembled, viewed independently by three qualified clinicians, on reviewing anonymised images. Any centres submitting photographs of inadequate standard will be offered advice and training.

The schedule of enrolments, interventions and assessments is summarised using the Standard Protocol Items: Recommendations for Interventional Trials (SPIRIT) schedule as Fig. 3; and the SPIRIT Checklist provided as Additional file 1.

\section{Safety and adverse event reporting}

Standard definitions and procedures for the management of adverse events (AE), serious adverse events (SAE), serious adverse reactions (SAR) and suspected unexpected serious adverse reactions (SUSAR) are adopted within the trial. AEs and reactions are reported using relevant Case Record Form (CRF), whether related to treatment or disease, within 28 days following the last trial treatment. This will be antibiotics and mouthwash in the control arm, and HBO in the treatment arm. In addition, a new diagnosis of cancer or 
Table 2 Outcome measures, time of assessment and method of assessment

\begin{tabular}{|c|c|c|}
\hline Outcome measure & Timing & Method of assessment \\
\hline \multicolumn{3}{|l|}{ Primary outcome } \\
\hline Presence of ORN & 6 months & $\begin{array}{l}\text { Blinded review of radiograph, clinical photograph with corroboration from site investigator's } \\
\text { assessment in CRF (and dimensions of any exposed bone) }\end{array}$ \\
\hline \multicolumn{3}{|l|}{ Secondary outcomes } \\
\hline Presence of ORN & 3 and 12 months & As primary endpoint, but without radiographs unless ORN clinically evident \\
\hline Severity of ORN & 3,6 and 12 months & $\begin{array}{l}\text { Blinded review of radiograph, clinical photograph, site investigator's assessment (and } \\
\text { dimensions of any exposed bone) using modified Notani Score }[23,24]\end{array}$ \\
\hline Quality of life & 3,6 and 12 months & University of Washington head and neck cancer questionnaire \\
\hline Pain following surgery & 3,6 and 12 months & Patient-reported on a Visual Analogue Scale and use of analgesia \\
\hline Acute symptoms & $\begin{array}{l}\text { Days } 1 \text { thru } 7 \text { post } \\
\text { surgery }\end{array}$ & Patient-reported Likert scale for pain, swelling, trismus, normalcy of diet \\
\hline $\begin{array}{l}\text { Assessment of implants } \\
\text { (where relevant) }\end{array}$ & At closure of trial & Casenote review, by loss of any implant placed as part of trial \\
\hline $\begin{array}{l}\text { Late follow-up of MBS cases } \\
\text { (where relevant) }\end{array}$ & At closure of trial & Casenote review, by severity of ORN using modified Notani Score $[23,24]$. \\
\hline $\begin{array}{l}\text { Safety of } \mathrm{HBO} \text { related to } \\
\text { cancer recurrence }\end{array}$ & $\begin{array}{l}\text { Within } 12 \text { months of } \\
\text { trial treatments }\end{array}$ & SAE reporting. \\
\hline Safety of $\mathrm{HBO}$, otherwise & Within 28 days of $\mathrm{HBO}$ & $\mathrm{AE} / \mathrm{SAE}$ reporting of symptoms related to hyperbaric treatment \\
\hline
\end{tabular}

$A E$ adverse event, CRF Case Record Form, HBO hyperbaric oxygen, MBS minor bone spicules, ORN osteoradionecrosis, SAE serious adverse event

recurrence of cancer will be reported as an SAE for the duration of the patient's involvement in the study up to 12 months after surgery, irrespective of whether this is related to head and neck cancer, or the original indication for radiotherapy.

\section{Statistical considerations}

\section{Sample size}

Assuming an ORN rate of $5 \%$ in the $\mathrm{HBO}$ arm at 6 months following surgery consistent with an earlier RCT [10], 103 evaluable patients per group would



Fig. 2 Criteria for diagnosis of osteoradionecrosis (ORN) in the Hyperbaric Oxygen for the Prevention of Osteoradionecrosis (HOPON) trial [24]. ORN, further classified [23]: Notani 1: ORN confined to alveolar bone. Notani 2: limited to the alveolar bone and/or above the level of the inferior alveolar canal. Notani 3: ORN under the lower part of the inferior alveolar canal, with fistula or bone fracture 


\begin{tabular}{|c|c|c|c|c|c|c|}
\hline Procedure & $\begin{array}{c}\text { Following } \\
\text { randomisation }\end{array}$ & $\begin{array}{l}\text { At time of } \\
\text { Surgery }\end{array}$ & $\begin{array}{c}\text { 3 months } \\
\text { following } \\
\text { surgery }\end{array}$ & $\begin{array}{l}6 \text { months } \\
\text { following } \\
\text { surgery }\end{array}$ & $\begin{array}{c}12 \text { months } \\
\text { following } \\
\text { surgery }\end{array}$ & $\begin{array}{c}\text { At closure of } \\
\text { Trial }\end{array}$ \\
\hline Orthopantomogram & $\mathrm{x}$ & & $\begin{array}{l}\text { Only in cases } \\
\text { where ORN } \\
\text { present }\end{array}$ & $\mathrm{X}$ & $\begin{array}{l}\text { Only in cases } \\
\text { where ORN } \\
\text { present }\end{array}$ & \\
\hline Clinical Photograph & $\mathrm{x}$ & & $\mathrm{x}$ & $\mathrm{x}$ & $\mathrm{x}$ & \\
\hline Clinical Assessment & $\mathrm{x}$ & & $\mathrm{X}$ & $\mathrm{x}$ & $\mathrm{x}$ & \\
\hline $\begin{array}{l}\text { Pain Assessment } \\
\text { Chart (Appendix 7) }\end{array}$ & $\mathrm{x}$ & & $\mathrm{x}$ & $\mathrm{x}$ & $\mathrm{x}$ & \\
\hline $\begin{array}{l}\text { UoW QOL v4 } \\
\text { Questionnaire } \\
\text { (modified) } \\
\text { (Appendix 6) }\end{array}$ & $\mathrm{x}$ & & $\mathrm{x}$ & $\mathrm{x}$ & $\mathrm{x}$ & \\
\hline $\begin{array}{l}\text { Assessment of } \\
\text { suitability for } \mathrm{HBO}\end{array}$ & $\mathrm{x}$ & & & & & \\
\hline $\begin{array}{l}\text { Acute symptoms } \\
\text { questionnaire } \\
\text { (Appendix 8) }\end{array}$ & & $\mathrm{x}$ & & & & \\
\hline Blood Sample & & $\begin{array}{l}\mathrm{X} \text { (or at any } \\
\text { time after } \\
\text { randomisatio } \\
\mathrm{n} \text { if not at the } \\
\text { time of } \\
\text { surgery) } \\
\end{array}$ & & & & \\
\hline $\begin{array}{l}\text { Assessment of } \\
\text { severity of } \\
\text { diagnosed } \\
\text { osteoradionecrosis } \\
\text { (where appropriate) }\end{array}$ & & & $\mathrm{x}$ & $\mathrm{x}$ & $\mathrm{x}$ & $\begin{array}{l}\mathrm{X} \text { (where MBS } \\
\text { present) }\end{array}$ \\
\hline $\begin{array}{l}\text { Assessment of } \\
\text { implants (where } \\
\text { appropriate) }\end{array}$ & & & & & & $\mathrm{x}$ \\
\hline
\end{tabular}

Fig. 3 Standard Protocol Items: Recommendations for Interventional Trials (SPIRIT) trial schedule

provide $80 \%$ power to detect an odds ratio of 0.23 (equivalent to detecting an absolute difference of $13.5 \%$ between treatment arms) at a 5\% two-sided significance level. An estimated dropout rate was extrapolated from feasibility data at $7 \%$, resulting in an estimate of 221 patients to be recruited in total.

\section{Analysis plan}

It is not planned to fully adhere to the intention-to-treat (ITT) principle of 'analysing as randomised' because patients do not have the intervention ( $\mathrm{HBO}$ or standard care) until they have been randomised and had surgery arranged. Feasibility data revealed a somewhat higher dropout rate after randomisation in the $\mathrm{HBO}$ arm because of the increased opportunity available in the time taken to schedule HBO. Furthermore, the clinical question to be answered is the effect of $\mathrm{HBO}$, but only among those receiving surgery, and not the effect of $\mathrm{HBO}$ in itself. Demographic and clinical factors for the HBO and control groups will be presented both 'as randomised' and also 'as receiving surgery'. A multiple logistic regression analysis will be performed to confirm that these factors remain jointly uninformative for receiving surgery.

The primary test of efficacy in terms of risk of ORN at 6 months will be carried out using an exact logistic regression including a fixed term for treatment arm. The null hypothesis is that inclusion of $\mathrm{HBO}$ treatment pre-surgery is not more effective than standard care alone; that is, the odds ratio is not statistically different from 1 , while the alternate hypothesis is that $\mathrm{HBO}$ treatment is superior to standard care with an odds ratio of 0.23 or less. The test will be two-sided and a $P$ value of less than 0.05 will be declared statistically significant. Two-sided $95 \%$ confidence limits for the odds ratio will be presented for consistency with the significance test. Significance tests for secondary endpoints will also be two-sided at 5\% accompanied by $95 \%$ two-sided confidence intervals.

\section{Interim analyses}

A feasibility stage was incorporated into the original funding application with endpoints of ability to recruit, randomise and complete trial protocols for 50 patients within 2 years of commencement. Following this, regular analyses of trial data by the Independent Safety Data Monitoring Committee (ISDMC) review occur at 12-monthly intervals to assess recruitment rates and toxicity. A single, formal interim analysis will be carried out when 100 dental extraction patients have been followed up to the primary endpoint at 6 months. The Peto stopping rule will be implemented for the primary efficacy outcome (ORN rate at 6 months following surgery), and a futility analysis will also be carried out.

\section{Sensitivity analyses}

These will consist of:

1. Assessing the effects of assuming that: 
(a) all pre-surgery dropouts would have developed ORN

(b)no pre-surgery dropouts would have developed ORN

(c) all pre-surgery dropouts would have developed ORN with risk for their arm

(d)all pre-surgery dropouts would have developed ORN with mean risk across both arms

2. Assessing the effect of adjustment in analysis of the primary outcome for radiotherapy dose, age, sex, tobacco and alcohol use

3. Inclusion of multiply imputed primary endpoint responses for all randomised patients

4. Comparison of results using the site principal investigator's (PI's) original assessment of ORN (as with the primary analysis, this will include assessment of centre effects)

5. Analysis of 'blinded review' ORN after including MBS vs excluding cases with only MBS

\section{Translational research}

In addition to the routine trial assessments, translational blood samples will be taken either at the time of surgery, or at any time after randomisation. Sample collection kits will be sent to all participating sites and the samples collected and stored to Good Clinical Laboratory Practice (GCLP) standard at the University of Liverpool. These samples will be used at a future date to discover/ evaluate potential biomarkers of the genomic determinants of ORN [25].

\section{Trial oversight and regulatory arrangements Trial Management Group (TMG)}

This comprises the chief investigator (CI), other lead investigators (clinical and non-clinical) and members of the LCTU. The TMG will be responsible for the day-today running and management of the trial and will meet approximately monthly.

\section{Trial Steering Committee (TSC)}

The TSC will consist of the TMG plus three independent members, two patient representatives and an independent chair. The TSC will provide overall supervision for the trial and provide advice through its independent chair. The ultimate decision for the continuation of the trial lies with the TSC.

Independent Safety Data Monitoring Committee (ISDMC) The ISDCM will be responsible for reviewing and assessing recruitment, interim monitoring of safety and effectiveness, trial conduct and external data. The ISDMC will first convene prior to trial opening and will then define frequency of subsequent meetings (at least annually). The ISDMC will provide a recommendation to the TSC concerning the continuation of the study.

\section{Sponsorship}

The HOPON trial is co-sponsored by the University of Liverpool and Aintree University Hospitals NHS Foundation Trust.

\section{Registration}

The HOPON trial is registered with the European Clinical Trials Database (EudraCT 2007-006225-27).

\section{Clinical trial authorisation}

The HOPON trial uses oxygen which is classified by the Medicines and Healthcare Products Regulatory Authority (MHRA) as an Investigational Medicinal Product. The trial has received a clinical trial authorisation by the MHRA under the Medicines For Human Use (Clinical Trials) Regulations 2004 S.I. 2004/1031 (MHRA reference: 04196/0010/001-0019).

\section{Discussion}

Several areas of controversy have arisen during the development of the HOPON protocol. The first of these is the need for blinding. Some comparable trials in other fields of late radiation toxicity have used a sham HBO arm with the aim of producing a double-blind trial [26$28]$. The sham $\mathrm{HBO}$ arm used in such trials varies considerably. One approach [26] utilises a breathing mixture of $9 \%$ oxygen and $91 \%$ nitrogen so that at 2.4 ATA, arterial $\mathrm{PaO}_{2}$ is similar to breathing $21 \%$ oxygen at ambient air pressure. This, however, still exposes patients to the documented risks of hyperbaric pressure, and is by some considered unethical because of the implied issues of safety without any chance of benefit. Another approach used $40 \%$ oxygen and $60 \%$ nitrogen [28] providing an alternative lower 'dose' of oxygen treatment and correlation of outcome rather than a true control arm. More recently [27, 29] sham $\mathrm{HBO}$ arm using air at 1.1 ATA [29] or 1.3 ATA [27] have been used to provide a minimal increase in partial pressure of oxygen $\left(\mathrm{PpO}_{2}\right)$ while providing the sensations of compression. Regardless of mode of delivery, the provision of sham HBO treatments are equivalent in cost to those delivered on the treatment arm which, given finite resources, may impact the cost-effectiveness of such research. Finally, it is also uncertain whether the HBO chamber staff could ever be effectively blinded as they remain in control of the chamber. It is, therefore, possible that patients become unblinded when treated for over 30 treatment sessions, particularly as these sham treatments are delivered at a regular and different daily session to the oxygen treatments. 
The need for a blinded trial using sham treatments is considered desirable in trials where the primary endpoint is subjective or employ patient-reported outcome measures. In the HOPON trial the primary outcome is the presence of ORN, which is considered more objective, and additionally can be assessed on clinical photographs and radiographs at a remote time and location, by a panel of investigators who are blinded to the treatment arm received. However, the unblinded trial design for HOPON does introduce the possibility of reporting bias in some of the more subjective secondary outcomes such as quality of life or pain. Further, the unblinded design of the trial and need for extra treatments in the $\mathrm{HBO}$ arm introduces an inequality in time between randomisation and surgery for that arm. This potential methodological concern means that the time points commence at the time of surgery, rather than the date of randomisation, where it might be argued that only at randomisation are the two groups truly comparable.

Another difficulty that has arisen during the feasibility stage of the trial is the difficulty in robust allocation of some early stages of ORN. These have subsequently been classified as minor bone spicules (MBS), but are not reflected within any of the classifications of ORN currently in use. This difficulty is presumably not unique to the HOPON trial, yet it is difficult to ascertain from other similar studies how such cases have been dealt with. This is a problem created by taking a working 'textbook definition' of a clinical condition and extrapolating it as a primary outcome measure in a clinical trial. Because of the frequent (19\%) finding of MBS during a blinded interim data review, it was logical to introduce an additional class to reflect this, the justification for which has been separately published [24]. To establish if any of the MBS cases in the HOPON trial will progress to clinically significant ORN, there is a secondary outcome measure of outcome of such patients at the conclusion of the trial. As a means to avoid any ambiguity, therefore, the use of the Notani classification with the additional category of MBS has been employed in order to avoid subjectivity and to enhance reliability and consistency of reporting.

Further concerns were around how to deal with missing data, dropouts and the effect of differing complexity and length of treatment between the two arms. While ITT analysis is usual practice for the primary analysis in superiority trials, in this trial 'analysing as randomised' would not answer the clinical question which relates to a fixed time following surgery, in other words the intended primary analysis is essentially on a per-protocol or treatment-received basis. While unusual, this is one of the circumstances in which, as Piantadosi [30] has pointed out, too strict adherence to the ITT principle is unhelpful or inappropriate.
The HOPON trial design has, therefore, evolved through analysis of feasibility data and through blinded interim data analysis. This is the only formally conducted, multi-centre, RCT conducted in this field that the authors are aware of, and it is the first since Marx's single-centre study [10] published over three decades ago. The trial will provide data on the incidence of ORN after dental extraction and implant placement as well as the effect of $\mathrm{HBO}$ on retention of implants. Importantly, HOPON will provide translational resource for future studies of the genomic determinants of ORN in a group of patients treated to a defined protocol.

\section{Trial status}

Feasibility and interim analysis complete. Patient recruitment and data collection is ongoing to Protocol 9, dated 11 January 2017.

\section{Additional file}

Additional file 1: SPIRIT 2013 Checklist: recommended items to address in a clinical trial protocol and related documents. (DOC $121 \mathrm{~kb}$ )

\begin{abstract}
Abbreviations
ATA: Atmospheres; GCLP: Good Clinical Laboratory Practice; HBO: Hyperbaric oxygen; HOPON: Hyperbaric Oxygen for the Prevention of

Osteoradionecrosis; IMRT: Intensity-modulated radiotherapy; ISDMC: Independent Safety Data Monitoring Committee; ITT: Intention-totreat; MBS: Minor bone spicules; MHRA: Medicines and Healthcare Products Regulatory Authority; NICE: National Institute for Health and Care Excellence; ORN: Osteoradionecrosis; PI: (Site) principal investigator; $\mathrm{PpO}_{2}$ : Partial pressure of oxygen; RCT: Randomised controlled trial; SPIRIT: Standard Protocol Items Recommendations for Interventional Trials; TSC: Trial Steering Committee
\end{abstract}

\section{Acknowledgements}

The HOPON Trial Management Group wish to acknowledge the efforts of the HOPON Independent Data Monitoring Committee: Mr. Jim Paul, Cancer Research UK. Clinical Trials Unit Glasgow; Mr. Jeremy McMahon, Southern General Hospital Glasgow; Dr. Gerry Robertson, Beatson West of Scotland Cancer Centre Glasgow; and the HOPON Trial Steering Committee: Mrs. Sarah Brown, Clinical Trials Research Unit, University of Leeds; Dr. Richard Simcock, Sussex Cancer Centre, Brighton; Dr. Mark Glover, Hyperbaric Medicine Unit, Chichester; Dr. Syed Hussain, Department of Molecular and Clinical Cancer Medicine, University of Liverpool; Mr. Dominic Macareavy and Mr. John Richardson (Independent Lay Members). In addition, we acknowledge Cancer Research UK for funding.

\section{Funding}

The HOPON trial feasibility study was funded by Cancer Research UK Feasibility Study Committee (ref C23033/A9397). The phase III trial is funded by Cancer Research UK Clinical Trial Awards and Advisory Committee (ref C23033/A12122). Additional funding for the excess treatment costs of $\mathrm{HBO}$ were provided by the National Health Service Subvention Fund.

Availability of data and materials

Full HOPON protocol available on request through corresponding author.

\section{Authors' contributions}

RJS, BT, MB, CB and SC co-wrote the HOPON protocol. PS and SD acted as trial statisticians. RJS is the $\mathrm{Cl}$ of the HOPON trial. MB and BT are HOPON trial co-ordinators. GS, PB, KW, JM, PN and AK contributed to the funding applications and influenced the design of the trial, also acting as site investigators in recruiting to the trial. All authors read and approved the final manuscript. 


\section{Authors' information}

Richard Shaw (corresponding author), Professor of Head and Neck/Oral and Maxillofacial Surgery, Mersey Head and Neck Oncology Research Group, CRUK Liverpool Cancer Trials Unit, Department of Molecular and Clinical Cancer Medicine, University of Liverpool and Aintree University Hospital NHS Foundation Trust. Christopher Butterworth, Consultant in Restorative Dentistry, CRUK Liverpool Cancer Trials Unit, Department of Molecular and Clinical Cancer Medicine, University of Liverpool, Aintree University Hospital NHS Foundation Trust and Liverpool University Dental Hospital. Binyam Tesfaye, CRUK Liverpool Cancer Trials Unit, Department of Molecular and Clinical Cancer Medicine, University of Liverpool. Matthew Bickerstaff, CRUK Liverpool Cancer Trials Unit, Department of Molecular and Clinical Cancer Medicine, University of Liverpool. Susanna Dodd, Department of Biostatistics, University of Liverpool. Gary Smerdon, Research Director, Devon Diseases Research Centre. Seema Chauhan, Operational Director, CRUK Liverpool Cancer Trials Unit, Department of Molecular and Clinical Cancer Medicine, University of Liverpool. Peter Brennan, Professor and Consultant in Oral and Maxillofacial Surgery, Portsmouth Hospitals NHS Trust; peter.brennan@porthosp.nhs.uk. Keith Webster, Consultant in Oral and Maxillofacial Surgery, Queen Elizabeth Hospital, University Hospitals Birmingham NHS Foundation Trust. James McCaul, Professor and Consultant in Oral and Maxillofacial Surgery, Queen Elizabeth University Hospital, Glasgow. Peter Nixon, Consultant in Restorative Dentistry. Anastasias Kanatas, Professor and Consultant in Oral and Maxillofacial Surgery Leeds Dental Institute. Paul Silcocks, Trials Statistician, CRUK Liverpool Cancer Trials Unit, Department of Molecular and Clinical Cancer Medicine, University of Liverpool.

\section{Ethics approval and consent to participate}

The HOPON trial protocol has been granted ethical approval by Greater Manchester Central Research Ethics Committee (REC reference 08/H1008/32). All patients have been consented under this approval.

\section{Consent for publication}

Patient consent included the publication of all data.

\section{Competing interests}

The authors declare that they have no competing interests.

\section{Publisher's Note}

Springer Nature remains neutral with regard to jurisdictional claims in published maps and institutional affiliations.

\section{Author details}

${ }^{1}$ CRUK Liverpool Cancer Trials Unit, Department of Molecular and Clinical Cancer Medicine, University of Liverpool and Aintree University Hospital NHS Foundation Trust, Liverpool, UK. ${ }^{2}$ CRUK Liverpool Cancer Trials Unit, Department of Molecular and Clinical Cancer Medicine, University of Liverpool, Liverpool, UK. ${ }^{3}$ Department of Biostatistics, University of Liverpool, Liverpool, UK. ${ }^{4}$ Devon Diseases Research Centre, Plymouth, UK. ${ }^{5}$ Portsmouth Hospitals NHS Trust, Portsmouth, UK. ${ }^{6}$ Queen Elizabeth Hospital, University Hospitals Birmingham NHS Foundation Trust, Birmingham, UK. ${ }^{7}$ Queen Elizabeth University Hospital, Glasgow, UK. ${ }^{8}$ Leeds Dental Institute, Leeds, UK.

Received: 26 May 2017 Accepted: 17 November 2017

Published online: 10 January 2018

\section{References}

1. Harris M. The conservative management of osteoradionecrosis of the mandible with ultrasound therapy. Br J Oral Maxillofac Surg. 1992;30(5):313-8.

2. Marx RE. A new concept in the treatment of osteoradionecrosis. J Oral Maxillofac Surg. 1983;41(6):351-7.

3. Store G, Boysen M. Mandibular osteoradionecrosis: clinical behaviour and diagnostic aspects. Clin Otolaryngol Allied Sci. 2000;25(5):378-84.

4. Dhanda J, Pasquier D, Newman L, Shaw R. Current concepts in osteoradionecrosis after head and neck radiotherapy. Clin Oncol (R Coll Radiol). 2016;28(7):459-66.

5. Kanatas AN, Rogers SN, Martin MV. A survey of antibiotic prescribing by maxillofacial consultants for dental extractions following radiotherapy to the oral cavity. Br Dent J. 2002;192(3):157-60.

6. Shaw RJ, Butterworth $\mathrm{C}$. Hyperbaric oxygen in the management of late radiation injury to the head and neck. Part II: prevention. Br J Oral Maxillofac Surg. 2011;49(1):9-13.
7. Nabil S, Samman N. Incidence and prevention of osteoradionecrosis after dental extraction in irradiated patients: a systematic review. Int J Oral Maxillofac Surg. 2011:40(3):229-43.

8. Schache AG, Powell NG, Cuschieri KS, Robinson M, Leary S, Mehanna H, Rapozo D, Long A, Cubie H, Junor E, et al. HPV-related oropharynx cancer in the United Kingdom: an evolution in the understanding of disease etiology. Cancer Res. 2016;76(22):6598-606.

9. Chang DT, Sandow PR, Morris CG, Hollander R, Scarborough L, Amdur RJ, Mendenhall WM. Do pre-irradiation dental extractions reduce the risk of osteoradionecrosis of the mandible? Head Neck. 2007;29(6):528-36.

10. Marx RE, Johnson RP, Kline SN. Prevention of osteoradionecrosis: a randomized prospective clinical trial of hyperbaric oxygen versus penicillin. J Am Dent Assoc. 1985;111(1):49-54.

11. Vudiniabola S, Pirone C, Williamson J, Goss AN. Hyperbaric oxygen in the prevention of osteoradionecrosis of the jaws. Aust Dent J. 1999. 44(4):243-7.

12. Wahl MJ. Osteoradionecrosis prevention myths. Int J Radiat Oncol Biol Phys. 2006;64(3):661-9.

13. Tong AC, Leung AC, Cheng JC, Sham J. Incidence of complicated healing and osteoradionecrosis following tooth extraction in patients receiving radiotherapy for treatment of nasopharyngeal carcinoma. Aust Dent J. 1999; 44(3):187-94.

14. Feldmeier JJ. Hyperbaric oxygen 2003: indications and results: the Hyperbaric Oxygen Therapy Committee report: Kensington. 2003.

15. Kanatas AN, Lowe D, Harrison J, Rogers SN. Survey of the use of hyperbaric oxygen by maxillofacial oncologists in the UK. Br J Oral Maxillofac Surg. 2005;43(3):219-25.

16. Rogers SN, Magennis P. Survey of fellows regarding a national study into treatment with hyperbaric oxygen. Br J Oral Maxillofac Surg. 2007; 45(5):433-5.

17. Dhanda J, Hall TJ, Wilkins A, Mason V, Catling J. Patterns of treatment of osteoradionecrosis with hyperbaric oxygen therapy in the United Kingdom. Br J Oral Maxillofac Surg. 2009;47(3):210-3.

18. Cancer of the upper aerodigestive tract: assessment and management in people aged 16 and over. https://www.nice.org.uk/guidance/NG36/chapter/ Recommendations-followup-of-people-with-cancer-of-the-upperaerodigestive-tract-and-management-of. Accessed 22 Dec 2017.

19. Granstrom G. Osseointegration in irradiated cancer patients: an analysis with respect to implant failures. J Oral Maxillofac Surg. 2005;63(5):579-85.

20. Shaw RJ, Sutton AF, Cawood JI, Howell RA, Lowe D, Brown JS, Rogers SN, Vaughan ED. Oral rehabilitation after treatment for head and neck malignancy. Head Neck. 2005;27(6):459-70.

21. Ryan P. RALLOC: Stata module to design randomized controlled trials. Stata Tech Bull. 2000;54:49-53.

22. US Navy: US Navy Treatment Table RN66. In: US Navy diving manual. Volume 5, Revision 6: Naval Sea Systems Command; 2011:20-46. http:// www.alohashoredivers.com/mwg-internal/de5fs23hu73ds/progress?id= p6GhYF8rqk-nLyDQIKrOQ583lsCHgGh5hYMNPVGXUL4

23. Notani K, Yamazaki Y, Kitada H, Sakakibara N, Fukuda H, Omori K, Nakamura M. Management of mandibular osteoradionecrosis corresponding to the severity of osteoradionecrosis and the method of radiotherapy. Head Neck. 2003;25(3):181-6.

24. Shaw $R$, Tesfaye B, Bickerstaff M, Silcocks P, Butterworth C. Refining the definition of mandibular osteoradionecrosis in clinical trials: The Cancer Research UK HOPON trial (Hyperbaric Oxygen for the Prevention of Osteoradionecrosis). Oral Oncol. 2017;64:73-7.

25. Ghazali N, Shaw RJ, Rogers SN, Risk JM. Genomic determinants of normal tissue toxicity after radiotherapy for head and neck malignancy: a systematic review. Oral Oncol. 2012;48(11):1090-100.

26. Annane D, Depondt J, Aubert P, Villart M, Gehanno P, Gajdos P, Chevret S. Hyperbaric oxygen therapy for radionecrosis of the jaw: a randomized, placebo-controlled, double-blind trial from the ORN96 study group. J Clin Oncol. 2004;22(24):4893-900.

27. Glover M, Smerdon GR, Andreyev HJ, Benton BE, Bothma P, Firth O, Gothard L, Harrison J, Ignatescu M, Laden G, et al. Hyperbaric oxygen for patients with chronic bowel dysfunction after pelvic radiotherapy (HOT2): a randomised, double-blind, sham-controlled phase 3 trial. Lancet Oncol. 2016;17(2):224-33.

28. Pritchard J, Anand P, Broome J, Davis C, Gothard L, Hall E, Maher J, McKinna F, Millington J, Misra VP, et al. Double-blind randomized phase II study of hyperbaric oxygen in patients with radiation-induced brachial plexopathy. Radiother Oncol. 2001;58(3):279-86. 
29. Clarke RE, Tenorio LM, Hussey JR, Toklu AS, Cone DL, Hinojosa JG, Desai SP, Dominguez Parra L, Rodrigues SD, Long RJ, et al. Hyperbaric oxygen treatment of chronic refractory radiation proctitis: a randomized and controlled double-blind crossover trial with long-term follow-up. Int J Radiat Oncol Biol Phys. 2008;72(1):134-43.

30. Piantadosi S. Clinical trials. A methodological perspective. Hoboken: Wiley; 2005.

Submit your next manuscript to BioMed Central and we will help you at every step:

- We accept pre-submission inquiries

- Our selector tool helps you to find the most relevant journal

- We provide round the clock customer support

- Convenient online submission

- Thorough peer review

- Inclusion in PubMed and all major indexing services

- Maximum visibility for your research

Submit your manuscript at www.biomedcentral.com/submit
Biomed Central 